\title{
Análisis contemporáneo de la filosofía económica en el contexto geopolítico latinoamericano'
}

Por César Augusto Arévalo Cuadra, Jairo Rojas Meza

Recibido: 20.07.2015 / Aprobado: 27.07.2015

“...Un pueblo sin pasado no tendrá presente ni futuro, al pasado se le supera pero no se le cancela."

Alejandro Serrano Caldera

\section{RESUMEN}

Latinoamérica sufrió un proceso de sincretismo trascendental en la cultura, la política, la economía, la institucionalidad y de manera relevante, en su ciencia y filosofía. Los americanos conviven con la reconstrucción de sus bases civilizatorias, como: el arte, la cultura e identidad. Pero, sobre todo con la necesidad de rescate de su filosofía y su ciencia. La geopolítica, ciencia encargada de la relación del estado y el espacio de interés político, con el objetivo de conseguir más influencia y mejor posicionamiento, direccionó acciones hacia Latinoamérica, queriendo instaurarse ideológicamente, valiéndose de las fracturas en el posicionamiento filosófico y científico regional para establecerse por medio de las ideas como un medio de dominación. Entre las corrientes prominentes de pensamiento contemporáneo que han ejercido influencia en la región, se encuentran: Propuesta de Desarrollo Capitalista, Propuesta de Desarrollo Socialista, Teología de la Liberación y se concluye con la Propuesta de Desarrollo Latinoamericano, seguidas de su contra argumento crítico. Sin embargo, la búsqueda del propio paradigma Latinoamérica sigue, y está determinado por la unión regional, la cual se vislumbra como el medio de cambio, integrador y a la vez, renovador del sistema social que permitirá un ejercicio geopolítico regional, identificado con la realidad y al servicio del desarrollo. Estos elementos siempre vivos en Latinoamérica, son importantes para mantener la senda de búsqueda de nuestra filosofía e identidad.

Palabras claves: Filosofía, económica, latinoamericano, corrientes de pensamiento, contemporáneo, geopolítica, paradigma, desarrollo.

\section{ABSTRACT}

Latin America underwent a process of syncretism that extended deep into the culture, politics, economics and institutions; but more relevant in its science and philosophy so. The Americans have had to live with the permanent reconstruction of their civilizational bases, such as its art, culture and identity. But above all the need to rescue their philosophy and science. Ceopolitics as a science responsible for the relationship of the state and space of political interest, in order to get more influence and better positioning, has directed its actions toward Latin America, practicing in the contemporary period ideological actions have wanted to establish using the fractures in the philosophical and scientific positioning of the region; to establish by means of ideas as a means of domination. Among the prominent global currents of contemporary thought that have had some influence in the region are: Proposal of Capitalist Development, Proposal for Socialist Development, Liberation Theology and concludes with the Proposal Latin American Development, followed by all critical argument against. But the pursuit of its own paradigm Latin America continues and is determined by the regional union, which is seen as the medium of exchange, integrator and innovator of the social system, which it will allow a regional geopolitical exercise, identified with reality and the service of the integral development. These elements always live in Latin America, are important to keep the path in search of our philosophy and identity.

Key words: Philosophy, economy, Latin American, thought currents, contemporary geopolitics, paradigm, development.

1 Artículo elaborado en el marco del Doctorado en ciencias Económicas de la Universidad Del Zulia de Maracaibo Venezuela por: César Augusto Arévalo Cuadra, Candidato a Doctor en Ciencias Económicas, Docente de la Facultad Regional Multidisciplinaria de Carazo - FAREM (Nicaragua) y Jairo Rojas Meza, Profesor Titular UNAN-MANAGUA. 


\section{Introducción}

$\mathrm{E}$ n el tiempo la Geopolítica ha tenido diferentes interpretaciones y definiciones, desde una ciencia determinante para la toma de decisiones en el nivel político, hasta una ciencia de carácter mítica. La geopolítica es una ciencia que se ha consolidado a través del tiempo, como conocimiento científico teórico y pragmático, que se inspira en la íntima relación entre el estado y el espacio de interés político (Garay, 2002). La geopolítica de los países se ha ido adaptando de acuerdo a las necesidades de la época, siempre con el objetivo de conseguir más zonas de influencia, más poder y más recursos naturales (Sánchez, 2012).

Dentro de los temas geopolíticos se encuentran aspectos que permiten interpretar los sucesos incidentes en las realidades: económicos, sociales e históricas, que determinan el derrotero de las transformaciones más del pensamiento latinoamericano contemporáneo en materia política, económica, social y religiosa.

El ensayo se enfoca en analizar el contexto geopolíticoylainfluenciadelascorrientesmásimportantes de pensamiento contemporáneo y su trascendencia en la región. Se hace una introducción a la temática con el ítem Filosofía Económica Latinoamericana, luego se continua analizando las corrientes de pensamiento más notables que han incidido sobre Latinoamérica, entre ellas se encuentran: Propuesta de Desarrollo Capitalista, Propuesta de Desarrollo Socialista, Teología de la Liberación y se concluye con la Propuesta de Desarrollo
Latinoamericano, todas seguidas de su contra argumento crítico.

Los espacios denominados crítica, están referidos a reflexiones sobre los matices contextuales latinoamericanos que han desfavorecido el desarrollo de estas corrientes de pensamiento en la región, siempre dentro del contexto geopolítico estratégico de cada corriente.

Debido al papel preponderante de la iglesia en la función geopolítica histórica y su proveniencia desde antes de nuestro tiempo, en este artículo se le confiere un apartado relacionado con el desarrollo del pensamiento en Latinoamérica como una forma de desarrollo intelectual y religioso latinoamericano, particularmente, en la intervención denominada teología de la liberación, por ser la de más realce contemporáneo en la intervención de la iglesia en la región.

\section{Desarrollo}

\section{Filosofía Económica Latinoamericana}

El filosofar latinoamericano aflora como consecuencia de un crecimiento nuevo y vigoroso, pero no puede asentarse en el terreno de la tradición propia. No puede reaccionar contra una filosofía vieja y caduca, porque esta filosofía desapareció junto con la Colonia.

Nacemos porque nuestra América ha crecido, ha evolucionado y como un resultado de este crecimiento,




empezamos a tomar conciencia de nosotros mismos, empezamos a filosofar, porque empezamos también a construir rascacielos, a fundar escuelas primarias, a hacer grandes negocios y a tener presupuestos estatales astronómicos. Pero, un negocio se puede realizar sin necesidad de pensar en ancestros; en cambio, filosofar sin una tradición, es imposible. La filosofía es comprensión espiritual y la comprensión espiritual sólo puede hacerse, aunque trascienda la historia, desde una perspectiva histórica (Vargas, 2010; 3).

El fenómeno de la destrucción de Las Civilizaciones Americanas, abarcó las expresiones: culturales, literarias, políticas, religiosas e institucionales. Sin embargo, el mayor daño que se le causó a esa nueva cultura sincrética, fue la destrucción de su filosofía y de su ciencia, dando paso a lo que se podría llamar la castración filosófica, porque el rumbo de la ciencia en sí, es el motor de la filosofía y este, el empuje general del desarrollo.

Durante muchos años la economía logró cierto grado de cientificidad en las sistematizaciones de conceptos, básicamente, el conocimiento científico es acumulativo, la economía y su técnica científica. En este campo, la técnica científica se ha confundido erróneamente con lo que es la ciencia económica, lo cual provocó grandes retrocesos en la formulación de nuevas teorías (Cerezo, 2010;16).

En Latinoamérica, a fines de la década de los 50 del siglo $\mathrm{XX}$ y durante las dos siguientes, las actividades de ciencia y tecnología se llevaron a cabo sobre la base del esfuerzo casi exclusivo del estado. Independientemente del hecho de que estos esfuerzos no provocaron una dinámica sostenida de innovación en el conocimiento y en la economía, predominó en muchos sectores el divorcio entre investigación y producción. Se desarrollaron dos modelos contiguos de investigación en ciencia y tecnología, con consignas y misiones claras y fuentes de legitimidad para sus funciones:

a. por una parte, la ciencia académica, basada principalmente en las universidades, es incorporada - aunque de manera periférica - a la comunidad científica internacional, de quien recibe su legitimidad, orientaciones y formas de organización, apoyándose en los criterios de calidad y excelencia. b. por otra parte, una actividad tecnológica, sustentada sobre todo, en organismos sectoriales y legitimada por un aparato de planificación estatal destinado a la resolución de problemas prácticos y a la transferencia de tecnologías al sector productivo o de defensa. Ambos, financiados por el estado, respondían, sin embargo, a lógicas diferentes.

Las categorías de superación filosófica de las distintas teorías económicas propuestas a través de modelos económicos enunciados para desarrollar a Latinoamérica se truncó, dando lugar a una secuencia de esfuerzos mal logrados.

El modelo de desarrollo económico que ha caracterizado a América Latina durante el siglo XX, presenta una evolución en el tiempo con varias etapas, cuyas características han sido el reflejo de opciones ideológicas diferentes siempre ligadas al devenir de la coyuntura internacional (Presbisch, 1981).

Ante la carencia de bases científicas y filosóficas, Latinoamérica se ve envuelta en un devenir de opciones teóricas económicas contrapuestas, las cuales tratan de establecer su desarrollo teórico, económico y filosófico, como baluarte de desarrollo latinoamericano para lograr mayor posicionamiento geopolítico.

El problema deviene en que ninguna logra apropiarse de las bases científicas y filosóficas latinoamericanas. Donde además surgen nuevas corrientes de pensamiento ajustadas a una realidad propia.

A continuación se exponen síntesis de la propuesta teórica económica de la corriente capitalista, con sus críticas, luego la realizada por la corriente socialista, continuando con la influencia de la iglesia y se cierra con la visión latinoamericana, todo en un marco contemporáneo.

\section{Propuesta de desarrollo capitalista y su crítica}

La teoría de sistema mundial capitalista, se centra en el estudio del sistema social y sus interrelaciones con el avance del capitalismo mundial, como fuerzas determinantes entre países. Wallerstein (1998) sugiere que la unidad de análisis no debe ser el estado-nación o la sociedad nacional, sino el sistema-mundo en su conjunto. 
Wallerstein define la economía-mundo capitalista como: "un sistema que incluye desigualdad jerárquica de distribución, basada en la concentración de producción (producción monopolizada y de alta rentabilidad), en zonas limitadas, que se convierten en fuente de acumulación de capital, que favorece el reforzamiento de las estructuras estatales. Plantea que el proceso de desarrollo es multidimensional.

Asimismo, analiza la formación y la evolución de los modos capitalistas de producción, como un sistema de relaciones económico - sociales, políticos y culturales, según Dos Santos (1998:130), "se distingue la existencia de un centro, una periferia y una semiperiferia, entre economías centrales, una economía hegemónica que articula al conjunto del sistema".

En este sistema las relaciones económicas del centro con los países semiperiféricos y periféricos dependen de tres factores estratégicos: el grado en que las industrias sean fundamentales en funcionamiento de cadenas de mercancías clave; el grado de importancia para sostener el nivel de demanda, efectiva para sectores de producción más rentables, y el grado de importancia en decisiones estratégicas (localización, poderío militar, materias primas, etc.).

La característica de desigualdad del sistemamundo, genera la apropiación de los excedentes de los países periféricos y semiperiféricos, por parte de los países avanzados. Esta expoliación se reproduce y se refuerza en el ámbito nacional y regional. El desarrollo de las fuerzas capitalistas influye en definir las relaciones de producción a nivel nacional y las características de operar en los países periféricos (Ortegón, 2000).

La evolución de este sistema dio lugar a las naciones, incluyendo las de tercer mundo y determinó su posición relativa en la jerarquía internacional. El enfoque va dirigido a variables como: los flujos comerciales entre naciones, ventajas geopolíticas relativas y fortalezas militares e ideológicas (Coria, 2008).

Este paradigma civilizatorio centrado en la creencia del posible crecimiento económico ilimitado en un planeta de recursos finitos, que identifica felicidad con riqueza, bienestar con acumulación de bienes materiales, progresoconconsumismo, en su desenvolvimiento teórico y modelístico, concluyó refutando el propio paradigma materializado en una profunda crisis internacional, que no se resume en la turbulencia financiera.

En consecuencia, todas las dimensiones de la vida sufren el acelerado proceso de mercantilización. El capitalismo reino del deseo infinito, se atasca en la paradoja de establecerse en un planeta finito, con recursos naturales limitados y una restringida capacidad poblacional (Bretto, 2012).

\section{Propuesta de desarrollo socialista y su crítica}

Diferentemente, en los países socialistas el objeto del fomento de la economía persigue la satisfacción de las crecientes demandas materiales y espirituales de la población, basada en la propiedad social sobre los medios de producción y el trabajo libre de explotación, desarrollo en consonancia con las leyes económicas objetivas del socialismo. La revolución científica técnica y el efecto que esta ejerce sobre la división social del trabajo, crean poderosas premisas materiales para la ampliación de los vínculos económicos entre las economías nacionales.

La preparación de los métodos científicos de planificación, estímulo y gestión de la producción, es la misión principal de la economía de la industria como una de las ciencias económicas.

La técnica es uno de los elementos principales de las fuerzas productivas de la sociedad, es el conjunto de los medios de producción que se emplean en el proceso de trabajo, para producir bienes materiales sobre los que recaen los procesos tecnológicos. La técnica constituye la base de la actividad productiva del hombre. El nivel técnico y sus proporciones en la industria, determinan las posibilidades de producción y los índices cuantitativos y cualitativos de la producción de la actividad industrial.

El progreso científico técnico es el más influyente de los factores en la creación de una estructura moderna de la industria. Este cambia las proporciones de las empresas y su distribución territorial, avanzan la especialización y la combinación de las unidades de la industria. Además, influencia sobre características cualitativas de la actividad de las empresas industriales sobre el empleo de los fondos fijos y circulantes, el mejoramiento de la calidad y la disminución sobre todos los factores del costo producto industria. 
Su influencia sobre todos los aspectos de la labor productiva y económica de las ramas y las empresas, hace indispensable la dirección a la creación y la puesta en servicio de los nuevos medios técnicos. La labor de dirección del actual progreso técnico comprende la labor de planificación, la estimulación económica, la organización y la gestión de la investigación y el empleo de los nuevos recursos técnicos, aspectos interconexos y realizables de manera integral. La labor de dirección del progreso técnico se expresa en primer término, en la planificación (Bacáev, 1981).

A pesar de las consideraciones anteriores, es necesario saber que la propuesta se llevará adelante en una realidad propia. La nueva sociedad en formación tiene que competir muy duramente con el pasado. Esto se hace sentir no solo en la conciencia individual, en la que pesan los residuos de una educación sistemáticamente orientada al aislamiento del individuo, sino también por el carácter mismo de este período de transición con persistencia de las relaciones mercantiles. La mercancía es la célula económica de la sociedad capitalista, mientras exista, sus efectos se harán sentir en la organización de la producción y, por ende, en la conciencia.

En el esquema de Marx se concebía el período de transición como resultado de la transformación explosiva del sistema capitalista destrozado por sus contradicciones. En la realidad posterior, se ha visto cómo se desgajan del árbol imperialista algunos países que constituyen ramas débiles, fenómeno previsto por Lenin. En estos, el capitalismo se ha desarrollado lo suficiente como para hacer sentir sus efectos de un modo u otro, sobre el pueblo, pero no son sus propias contradicciones las que, agotadas todas las posibilidades, hacen saltar el sistema.

La lucha de liberación contra un opresor externo, la miseria provocada por accidentes extraños, como la guerra, cuyas consecuencias hacen recaer las clases privilegiadas sobre los explotados, los movimientos de liberación destinados a derrocar regímenes neocoloniales, son los factores habituales de desencadenamiento. La acción consciente hace el resto.
En estos países no se ha producido todavía una educación completa para el trabajo social y la riqueza dista de estar al alcance de las masas, mediante el simple proceso de apropiación. El subdesarrollo por un lado y la habitual fuga de capitales hacia países «civilizados» por otro, hacen imposible un cambio rápido y sin sacrificios.

Resta un gran tramo a recorrer en la construcción de la base económica y la tentación de seguir los caminos trillados del interés material, como palanca impulsora de un desarrollo acelerado, es muy grande.

Se corre el peligro de que los árboles impidan ver el bosque. Persiguiendo la quimera de realizar el socialismo con la ayuda de las armas melladas que nos legara el capitalismo (la mercancía como célula económica, la rentabilidad, el interés material individual como palanca, etcétera), se puede llegar a un callejón sin salida. Y se arriba allí, tras de recorrer una larga distancia en la que los caminos se entrecruzan muchas veces y donde es difícil percibir el momento en que se equivocó la ruta. Entre tanto, la base económica adaptada ha hecho su trabajo de zapa sobre el desarrollo de la conciencia. Para construir el comunismo, simultáneamente con la base material hay que hacer al hombre nuevo (Guevara, 1965).

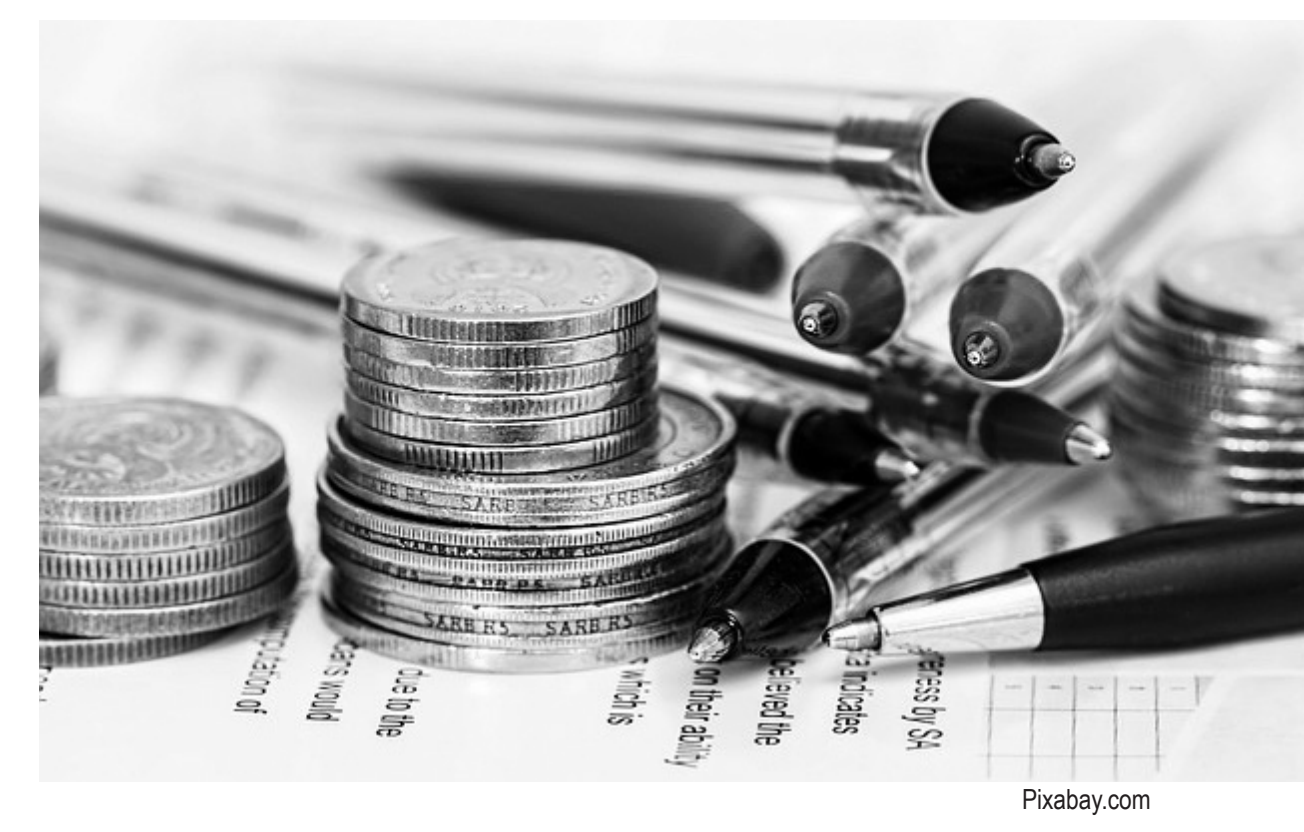




\section{Teología de la Liberación y su crítica}

La Teología de la Liberación, movimiento teológico contemporáneo, pretendió ser una respuesta a la necesidad de Solidaridad con los pobres. Es difícil definir la Teología de la Liberación, dado sus complejos y múltiples puntos de vista, pero puede decirse que busca analizar el significado de las clases sociales, la pobreza y la justicia. Una corriente bastante amplia de dicha teología, mezcla el marxismo y otras ideologías políticas con el cristianismo (Rivero, 1998).

En este mundo de pecado la autoridad de Dios es una bendición, la libertad sin Autoridad es una maldición. Se debe obedecer a las autoridades que nos gobiernan (Romanos 13). Esto significa que la subversión o la obstaculización de las funciones de los que gobiernan, como la Teología de la Liberación preconiza, es contrario a la voluntad de Dios.

$\mathrm{Si}$ el pueblo manda es soberano. Groen Van Prinsterer, señaló que la soberanía del pueblo significa que los organismos gobernantes con el fin de asegurar su propia conservación, tenderán de un modo u otro a una centralización del poder en nombre del bien común. De no ser así, la función de gobernar se convertirá en una tarea imposible. En esta estructura básica, en la realidad no existe una diferencia fundamental entre la democracia y el marxismo.

Tanto la democracia como el comunismo tienen como base fundamental el servicio al hombre y no al Señor; y para el hombre no existe una norma constante de justicia y libertad. La verdadera justicia y libertad son posibles cuando un gobierno se considera siervo de Dios. En análisis crítico realizado a la Teología de la Liberación, la búsqueda de la renovación del mundo de una manera secular, a través del marxismo o cualquier otra forma, está destinada al fracaso (Van Dan, 1996).

El mundo religioso, al igual que el político, está lleno de mentiras bien contadas. Se afirma la condena de la Teología de la Liberación por parte de Juan Pablo II, lo cual no es real. La Teología de la Liberación incluía en su agenda temas como la deuda externa, el capitalismo, los derechos de los pobres. Los pronunciamientos sobre cuestión social, venidos del máximo representante de la iglesia (El Papa), tratan críticamente los mismos temas.
Juan Pablo II nunca rechazó mediar en conflictos internacionales, guerras y masacres. Wojtila salió por el mundo adelante en peregrinación en pro de los valores de justicia social, familia y globalización de la solidaridad. En un mundo que exalta como valor la competitividad, la palabra es incómoda, para un sistema que se basa en la apropiación privada de la riqueza, y aliento a la esperanza de millones de seres humanos excluidos de los derechos humanos (Bretto; 2003).

La Teología de la Liberación acompañó el proceso latinoamericano de emancipación del capitalismo de EstadosUnidosy delordeneconómicoactual.Sinembargo, su valor fue parcial y recortado como aporte, porque en primer lugar la iglesia, a pesar de usarlo como un medio de balanza geopolítica para perpetuar su influencia en el mundo y particularmente, en Latinoamérica, no lo soltó del todo como medio de desarrollo del pensamiento latinoamericano, ni mucho menos aprovechó los réditos de la conservación y transformación, necesarios en todo proceso de crecimiento intelectual que pudiera haber en Latinoamérica, producto de la crisis sistémica.

Simplemente se limitó a "manejarlo" como un instrumento de negociación para decirle a los dominadores, tengo en mi seno el baluarte del cambio social, no tengo por qué defender ad honorem la supremacía del poder militar, y mi influencia es determinante en el balance de las fuerzas y en el desgaste del poder.

\section{Propuesta de desarrollo latinoamericano y su crítica}

Los modelos de desarrollo surgen como alternativa para re-conformar debilidades estructurales entre países, dentro del contexto latinoamericano destaca la integración económica.

La Comisión Económica para América Latina (CEPAL) estudia estos temas de interés económico. La contribución de la CEPAL a la historia del pensamiento económico, debe considerarse a partir del "reconocimiento, que se trata de un cuerpo analítico específico, aplicable a condiciones históricas propias de la periferia latinoamericana". El objetivo es combinar un método en esencia histórico e inductivo, con la referencia abstracto-teórica, que constituye la teoría Cepalina del subdesarrollo periférico latinoamericano (Bielschowsky, 1998; 10). 


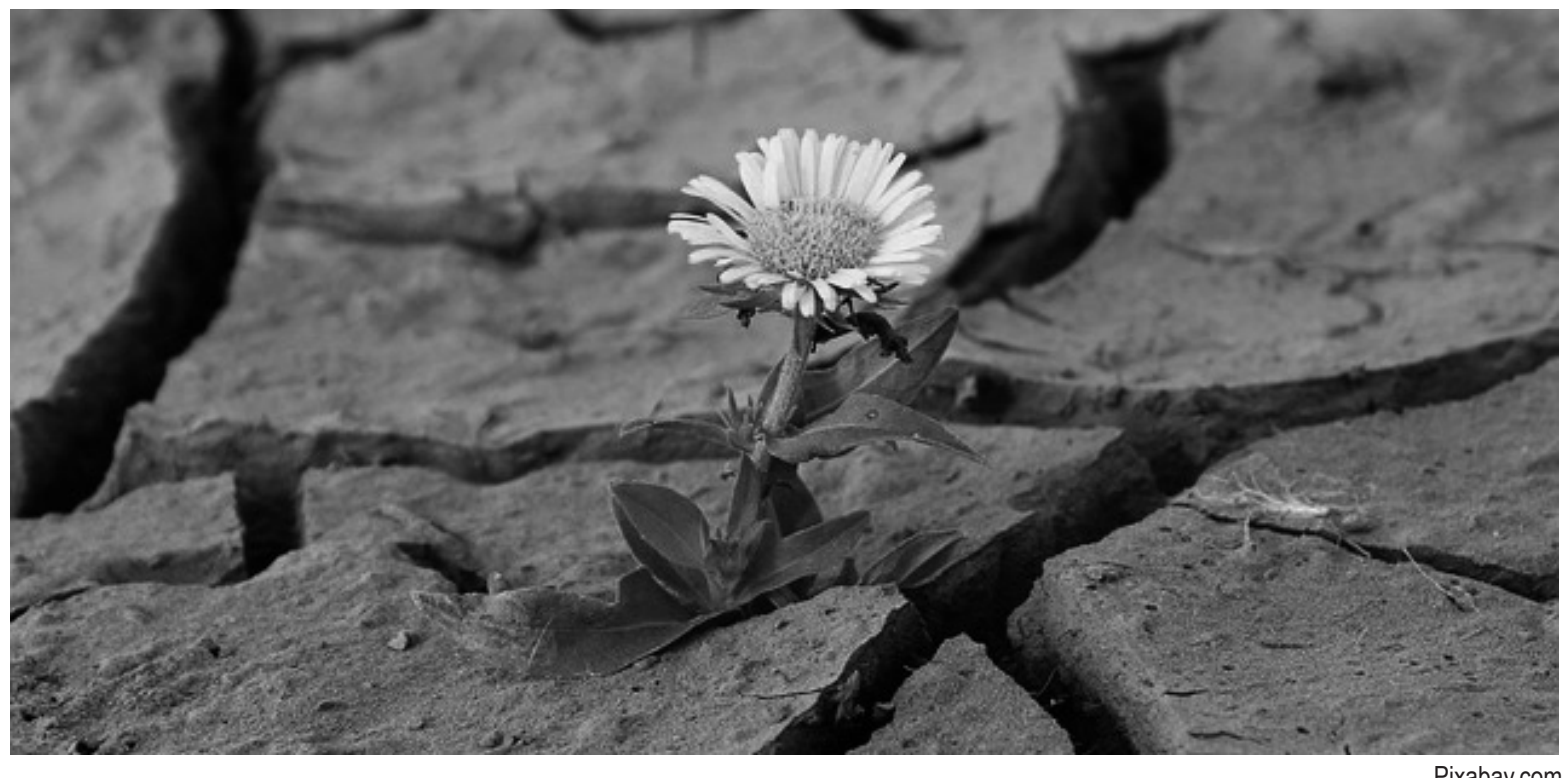

La integración latinoamericana ha sido búsqueda incansable de innumerables hombres que han luchado por ella en el transcurso de la historia. La unidad política deseada por Bolívar y Martí, a pesar de cambios significativos en la región desde fines del siglo $\mathrm{XX}$ y principios del XXI, no se ha logrado aún.

En el siglo $X X$ se hicieron intentos de lograr la integración económica en América Latina, desafortunadamente fracasaron, por no estar las condiciones objetivas creadas en algunos países y por no contar en otros, con los líderes ni organizaciones que respondieran realmente a las necesidades de los pueblos.

Esto en vínculo directo con la relación establecida entre países desarrollados y subdesarrollados, entre las que se presentan como limitantes:

1. El grado de conocimiento científico y técnico (tecnología moderna), no se adapta a las condiciones y dotación de factores en los países subdesarrollados.

2. Los modelos de organización e instituciones, no se adaptan a las necesidades del mundo subdesarrollado.

3. La ayuda gubernamental obstruye el progreso hacia el desarrollo, al apoyar y sostener regímenes feudales o conservadores, no dispuestos a realizar reformas sociales y políticas que el progreso demanda.
En Latinoamérica la conformación centro periferia devenida del "natural" proceso de conformación capitalista, trajo consigo la profundización de las debilidades estructurales (Seer, 1975; 38-34).

\section{Conclusiones}

En Latinoamérica se vivió un proceso complejo de sincretismo, que cercenó las bases del desarrollo científico, filosófico, económico, religioso, institucional y cultural; ha sido una preocupación válida de los americanos restablecer esas bases e integrarlas en una nueva cultura. Sin embargo, este crecimiento no ha sido fácil.

Las dificultades se han generado por la influencia que quieren ejercer sobre los pueblos latinoamericanos, las distintas corrientes de pensamiento, las cuales en la época contemporánea han tenido principalmente tres fuentes externas y una endógena.

La corriente capitalista ha promovido el desarrollo económico con modelos de mercado, tendientes a garantizar crecimiento económico acelerado, ha logrado enormes avances en cuanto a crecimiento económico, pero no ha sabido establecerse como una ideología latinoamericana propia.

Entre las principales dificultades para lograr aceptación y consenso regional, está el hecho de que se 
ha alcanzado crecimiento a costa de la sobre explotación de los recursos naturales, y que las grandes utilidades de ese aprovechamiento han quedado fuera de la región.

Existe también certeza de que el desarrollo del capitalismo ha establecido diferentes estratos de países y mientras unos se aprovechan de los recursos, a otros nos queda solo el despojo. Lo más crítico de este sistema económico, filosófico, institucional y cultural capitalista, es que estamos presenciando su agotamiento. Debido a la premisa de continuar creciendo a costa del sobre aprovechamiento de los recursos, lo que está conduciendo al mundo a un colapso.

En la otra acera Latinoamérica contemporánea, ha sido influenciada por la propuesta de desarrollo socialista, la cual tiene como premisa el crecimiento planificado de la economía, que se plantea garantizar a la población el abastecimiento necesario para desarrollase, por medio de un mejor uso de la ciencia, la técnica y la investigación.

La motivación característica fundamental de esta propuesta radica en que también habrá un desarrollo espiritual más acorde a las necesidades integrales del hombre, dado una vez más por la comprensión en toda su dimensión de los avances científicos técnicos al servicio de la sociedad. De manera que la justa compensación y distribución de la riqueza con el uso y apropiación social del conocimiento, conducirán a un pueblo mejor preparado para desarrollar el futuro.

Sin embargo, esta situación no ocurrió, porque como afirmó Ernesto Che Guevara, no existe en Latinoamérica las bases educativas y de desarrollo humano, necesarias para emprender esta marcha. Por lo que el desempeño de la propuesta ha tenido alcances limitados.
La Teología de la Liberación, pretende ser una respuesta a la necesidad de Solidaridad con los pobres, esta corriente teológica mezcla el marxismo y otras ideologías políticas con el cristianismo y es criticada por buscar la renovación del mundo de una manera secular, por encima de la supremacía de poder Religioso.

Por parte del liderazgo eclesial existe una falsa condena de la Teología de la Liberación y su notable vínculo en conflictos internacionales y sus principios e ideales como líder religioso.

Se ha utilizado la corriente de pensamiento como medio de negociación geopolítico, como un mandato o un permiso, según como se quiera ver, para recriminar al poder, en aras del balance; sin embargo no se le sacó el verdadero provecho como medio liberalizador del pensamiento para pronunciarnos de manera más acertada sobre nuestro derrotero futuro.

Por último, no se puede observar la panorámica latinoamericana sin considerar a la CEPAL, como vitrina de las consideraciones y propuestas de pensamiento más avanzado en Latinoamérica. Así como de sus contradicciones más profundas.

En el territorio existe una plena identificación entre países, y una claridad de la situación de fondo, donde se hace muy difícil sobrellevar una realidad impactada desde muy temprano por un proceso sincrético trascendental.

En donde es vista la integración regional como el paradigma del desarrollo territorial, que no se ha concretado por las mismas contradicciones intrínsecas de nuestros propósitos.

Debemos pues, vivir un proceso de crecimiento social y desarrollo humano que nos permita alcanzar nuestro objetivo y de esta manera transformar la realidad, el cual hará posible el crecimiento justo y equitativo de una importante región que es más grande de lo que la dejamos ser. 


\section{Bibliografía}

Bacáev, Y. (1981). Economía de la Industria Socialista (Traducido por L. Vládov). Moscú: Progreso.(p 13,16,145,148) (Original publicado en 1980.)

Bielschowsky, R. (1998). “Cincuenta años del pensamiento de la CEPAL: una reseña", en Cincuenta años de pensamiento en la CEPAL. Textos seleccionados, vol. 1, Fondo de Cultura Económica, CEPAL, Santiago, Chile, 1998, p. 10

Bretto, F. (2003). Juan Pablo II, Papa Político. Consultado en Abril 10-2012 en http://alainet.org/active/ show text_pt.php3?key $=4772$.

Bretto, F. (2012). Dictadura Económica. Consultado en marzo 30-2012 en http:/ / alainet.org/active/52558.

Cerezo, L. (2012). ¿Existe una Epistemología Latinoamericana? Caracas 2012, p.16

Coria, L. (2008). Teoría del sistema-mundo. Consultado en Marzo 21-2012 en http://www.eumed.net/ libros/2008a/344/Teoria \%20del\%20sistema\%20 mundo.htm.

Garay, R. (2002). Qué es Geopolítica? Consultado en Abril 13-2012 en http://meltingpot.fortunecity.com/ alberni/698/revista_docente/iv_vii/e5.html.

Guevara, E. (1995). El socialismo y el hombre en cuba. Seminario uruguayo 1965
Ortegón, J. (2000). La Globalización. Consultado en Marzo 28-2012 en http://www.ugt.es/globalizacion/ ortegon.htm

Presbisch, R. (1981).Capitalismo Periférico: Crisis y transformación.

Rivero, J. (1998). Teología de la liberación. Consultado en Abril 10-2012 en http://www.corazones.org/ apologetica/practicas/teologia_liberacion.htm.

Sánchez, A. (2012). Análisis de las Geopolítica Mundial del siglo XIX hasta Hoy. Consultado en Abril 112012 en http://es.scribd.com/ albertosanchez 29/d/79769756-analisis-de-la-geopoliticamundial-desde-el-siglo-xix-hasta-hoy\#download.

Santa Biblia. Romanos 13. Reina Valera - 1960, Sociedades Bíblicas Unidas.

Seer,D. Joy,L. (1975). El Desarrollo de un Mundo Dividido (Traducido por Suárez, E) PenguinBooks, Londres (p 38-44). Fondo de la Cultura Económica/ Contemporánea.(Original Publicado en 1971)

Van Dam. (1996). Teología de la Liberación (3ra Edicion). Consultado en Abril 02-2012 en http://www. iglesiareformada.com/Van_Dam La_ teologia liberacion.pdf

Vargas, G. (2010). El debate sobre la identidad de la filosofia. P; 3.consultado en http://csh.izt.uam. $\underline{\mathrm{mx} / \mathrm{cen} \_d o c / c e f i l i b e / \text { esbozo/07\%2520 }}$ debate $\%$ 252 0fil\% 2520lat.pdf

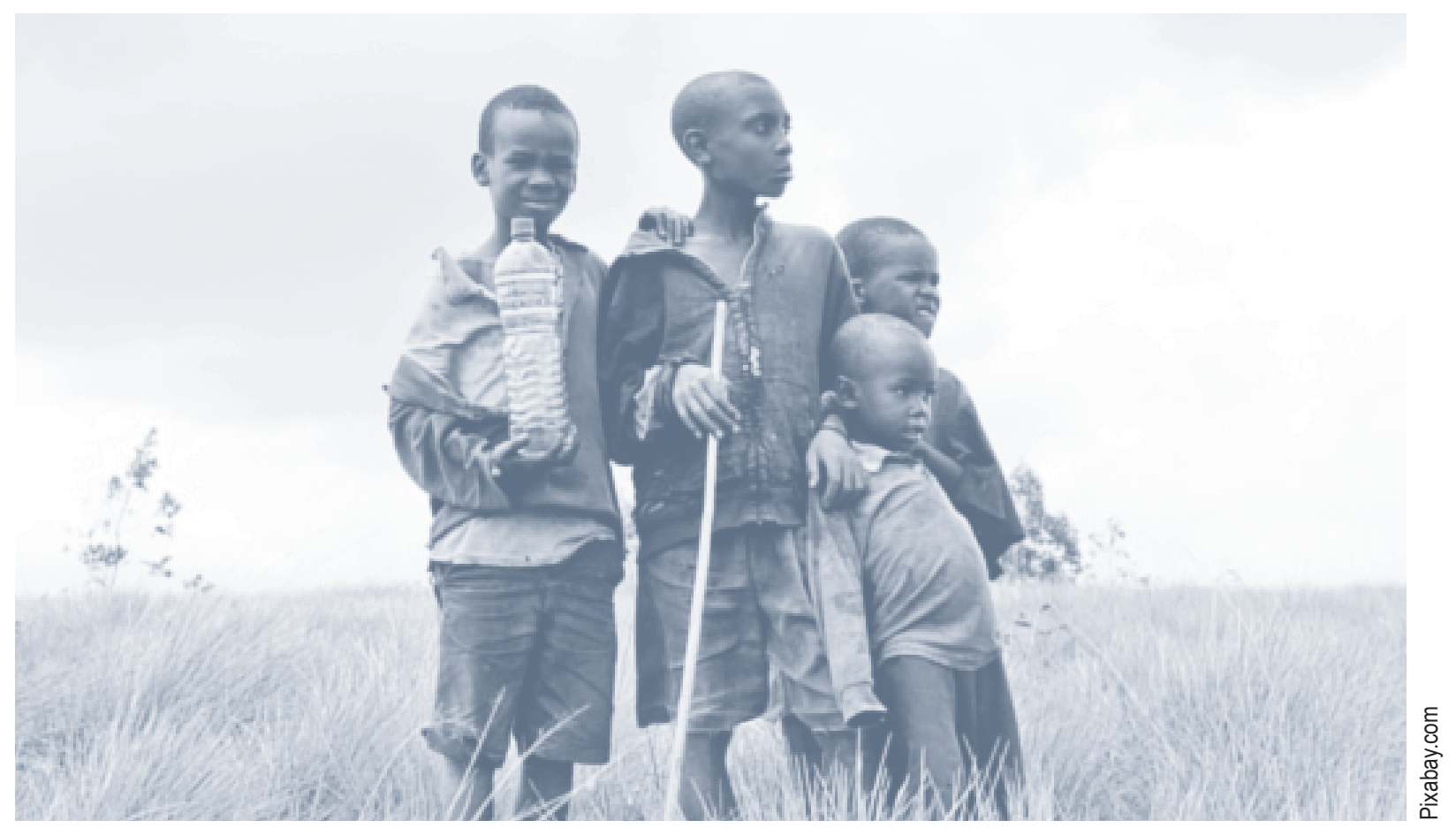

